\title{
Individual and Combined Effects of the Extractant, Surfactant and Modifier Concentrations on the Droplet Coalescence Time of the Primary Emulsion in the Liquid Surfactant Membrane Extraction Process
}

\author{
Adriane Salum, Lorena da Costa Nascimento, Tânia Lúcia Santos Miranda, \\ Leandro Dornellas Sampaio Andrade, Cibele Konzen, Julio Cézar Balarini, \\ Estêvão Magno Rodrigues Araújo, Fabrício Eduardo Bortot Coelho \\ Department of Chemical Engineering, Federal University of Minas Gerais, Belo Horiozonte, Brazil \\ Email: salum@deq.ufmg.br
}

How to cite this paper: Salum, A., da Costa Nascimento, L., Miranda, T.L.S., Andrade, L.D.S., Konzen, C., Balarini, J.C., Araújo, E.M.R. and Coelho, F.E.B. (2018) Individual and Combined Effects of the Extractant, Surfactant and Modifier Concentrations on the Droplet Coalescence Time of the Primary Emulsion in the Liquid Surfactant Membrane Extraction Process. Advances in Chemical Engineering and Science, 8, 205-214.

https://doi.org/10.4236/aces.2018.84014

\section{Received: July 23, 2018}

Accepted: September 10, 2018

Published: September 13, 2018

Copyright (๑) 2018 by authors and Scientific Research Publishing Inc. This work is licensed under the Creative Commons Attribution International License (CC BY 4.0). http://creativecommons.org/licenses/by/4.0/

\begin{abstract}
In this work, the individual and combined effects of the extractant, surfactant and modifier concentrations on the droplet coalescence time of the primary emulsion in the liquid surfactant membrane extraction process were evaluated, through emulsification experiments. Adogen 464 was used as extractant (carrier), and Escaid 110, as diluent. Two systems were studied. The first one composed by the extractant, the surfactant and the diluent, and the second one composed by the same reagents, but with the addition of 1-decanol as modifier. It was observed that, when the modifier is not present in the membrane phase, the surfactant not only stabilizes the primary emulsion, but, apparently, it also plays a role similar to that of the alcohol, promoting the solvation of the amine in a low polarity diluent. Furthermore, the extractant, a quaternary amine, helps to stabilize the primary emulsion in systems without a modifier. For membrane phases consisting of 1 or $5 \% \mathrm{w} / \mathrm{w}$ of Adogen 464 and $2 \%$ or $5 \%$ w/w of ECA 4360 , a concentration of $3 \% \mathrm{w} / \mathrm{w}$ of 1-decanol was sufficient to promote the solvation of Adogen 464 in Escaid 110 and to obtain a low droplet coalescence time.
\end{abstract}

\section{Keywords}

Liquid Surfactant Membranes, Emulsification, Adogen 464, ECA 4360, 1-Decanol, Droplet Coalescence Time 


\section{Introduction}

Separation by liquid surfactant membranes (LSM) has attracted considerable interest since its development by N. N. Li [1] due to its great potential application in industrial separation processes. LSM are an effective method for the selective separation and concentration of various species from different solutions, even in high dilution conditions. LSM are applied in metallic ions extraction, as copper [2] and gadolinium [3], nuclear materials like uranium [4], weak acids as citric acid [5], and in the treatment of liquid effluents, as in the removal of phenol [6].

The formation of these membranes is based on the theory of multiple emulsions. There are two possible phase configurations for the LSM system: aqueous/organic/aqueous $(\mathrm{W} / \mathrm{O} / \mathrm{W})$ or organic/aqueous/organic $(\mathrm{O} / \mathrm{W} / \mathrm{O})$. Independently of the configuration, the three phases are: receiving or internal or inner phase (IP), which receives the solute extracted from the external phase; membrane phase (MP), which is responsible for the selective permeation of the solute from the feed phase to the inner phase, and donor or outer phase, also called feed or external phase (EP), which contains the desired solute.

The LSM process basically consists of four steps: emulsification, permeation, settling, and deemulsification [7] [8] [9].

In the emulsification step, the primary emulsion is formed by the dispersion of the IP in the continuous MP, under intense stirring. In the permeation step, the primary emulsion is dispersed in the EP under mild stirring, forming a multiple emulsion. In this step, the solute is extracted from the EP to the MP and stripped to the IP as well. In the next step, the solute-enriched primary emulsion is separated from the exhausted EP by settling. Finally, in the deemulsification step, the primary emulsion is broken up, resulting in the separation of the IP enriched with the solute of interest from the MP.

In emulsion separation processes, such as the LSM, knowing the formation, stability, and break-up phenomena is extremely important. The conditions to obtain an emulsion depend on the facility of dispersing one liquid in the other. As the interfacial tension between two immiscible liquids is always larger than zero, the formation of emulsions greatly increases the surface area between the liquids, resulting in a corresponding increase in the system free interfacial energy. The emulsion obtained is therefore thermodynamically unstable and the dispersed droplets tend to coalesce to reduce their free energy. To increase the stability of the LSM system, a tensoactive agent, called a surfactant or emulsifier, is added to the MP to reduce the interfacial tension between the liquids and the dispersed phase coalescence rate through the formation of a steric barrier around them [10] [11]. Thus, the MP is composed by the surfactant and by the solvent responsible for the extraction of the solute.

In the present work, polyamine ECA 4360 was used as a tensoactive reagent and the solvent was composed of quaternary amine Adogen 464 (methyltrialkylammonium chloride) solvated in an aliphatic diluent (a kerosene), Escaid 110. As quaternary amines are generally little soluble in aliphatic diluents [12], the im- 
provement of the extractant solvation in Escaid 110 by the addition of a long chain alcohol to the solvent (1-decanol) was evaluated. This was the chosen system because it will be used in a further study for the phenylalanine extraction by liquid surfactant membranes.

The main goal of the present work was to evaluate, through emulsification experiments, the individual and combined effects of the extractant, surfactant and modifier concentrations in the liquid surfactant membrane extraction process. The droplet coalescence time of the primary emulsion was used as a response variable.

\section{Experimental}

\subsection{Materials}

All reagents were used without further purification. For the preparation of the IP solutions, analytical grade potassium chloride (Labsynth) and lithium carbonate (QM Química Moderna) salts were used. Adogen 464 (Sherex), a methyltrialkylammonium chloride was used as extractant; polyamine ECA 4360 (Exxon), density $0.9213 \mathrm{~g} / \mathrm{mL}$, viscosity $97 \mathrm{cSt}$ at $100^{\circ} \mathrm{C}$, mass fraction $2.13 \%$ nitrogen, as surfactant; Escaid 110 (Exxon), a mixture of hydrogenated aliphatic hydrocarbons with density between $0.786 \mathrm{~g} \cdot \mathrm{mL}^{-1}$ and $0.815 \mathrm{~g} \cdot \mathrm{mL}^{-1}$ at $20^{\circ} \mathrm{C}$ to $24^{\circ} \mathrm{C}$ and viscosity of $(1.4$ to 2.0$) \times 10^{-6} \mathrm{~m}^{2} \cdot \mathrm{s}^{-1}$ at $37.8^{\circ} \mathrm{C}$, as diluents; and 1-decanol (Merck-Schuchardt), as modifier. The emulsions were prepared in a homogenizer and broken up in an electrostatic coalescer, at $5 \mathrm{kHz}, 1.7 \mathrm{kV}$, and current lower than $1 \mathrm{~mA}[13]$.

\subsection{Experimental Procedure}

The discontinuous emulsification tests were conducted, considering the system composition defined for the separation of phenylalanine from synthetic solutions in a further study. The MP was prepared using Adogen 464, ECA 4360, and Escaid 110 in the concentrations presented in Table 1 . In another set of experiments, 1-decanol was used as a modifier. The IP was a $\mathrm{KCl}$ solution $\left(2 \mathrm{~mol} \cdot \mathrm{L}^{-1}\right)$ containing $\mathrm{Li}_{2} \mathrm{CO}_{3}$ with $\mathrm{pH}$ adjusted to 9.5.

The primary emulsions were prepared by slowly pouring the IP into an emulsification vessel containing the MP, under stirring. The mixture was stirred at 13,000 $\mathrm{min}^{-1}$, for $5 \mathrm{~min}$. The time was measured from the start of the addition of the IP. Afterwards, the primary emulsion was taken to the electrostatic coalescer for break-up and the coalescence times of the phases were recorded (Figure 1).

The investigated and the fixed parameters, as well as the operating levels carried out, are presented in Table 1 . The tests were conducted using the one-factor-at-a-time approach. To evaluate the individual and combined effects of the extractant, surfactant, and modifier concentrations in the emulsification step, the coalescence time of the IP droplets was measured. It is important to point that the primary emulsions should be sufficiently stable during the extraction process by liquid surfactant membranes, but they should present low droplet 
Table 1. Emulsification experiments conditions.

\begin{tabular}{cc}
\hline Investigated Parameters & Levels \\
\hline Extractant mass fraction (\%) & $2.0,3.0,5.0,7.0$ \\
Surfactant mass fraction (\%) & $0.5,1.0,3.0,5.0$ \\
Modifier mass fraction (\%) & $0,3.0,5.0,7.0$ \\
Fixed Parameters & Levels \\
Stirring speed $\left(\mathrm{min}^{-1}\right)$ & 13,000 \\
Stirring time $\left(\mathrm{min}^{-1}\right)$ & 5 \\
pH of the inner phase & 9.5 \\
$\mathrm{KCl}_{\mathrm{IP}}$ concentration $\left(\mathrm{mol} \cdot \mathrm{L}^{-1}\right)$ & 2.0 \\
Volume ratio $(\mathrm{MP} / \mathrm{IP})$ & $3: 1$ \\
Temperature $\left({ }^{\circ} \mathrm{C}\right)$ & 25 \\
\hline
\end{tabular}

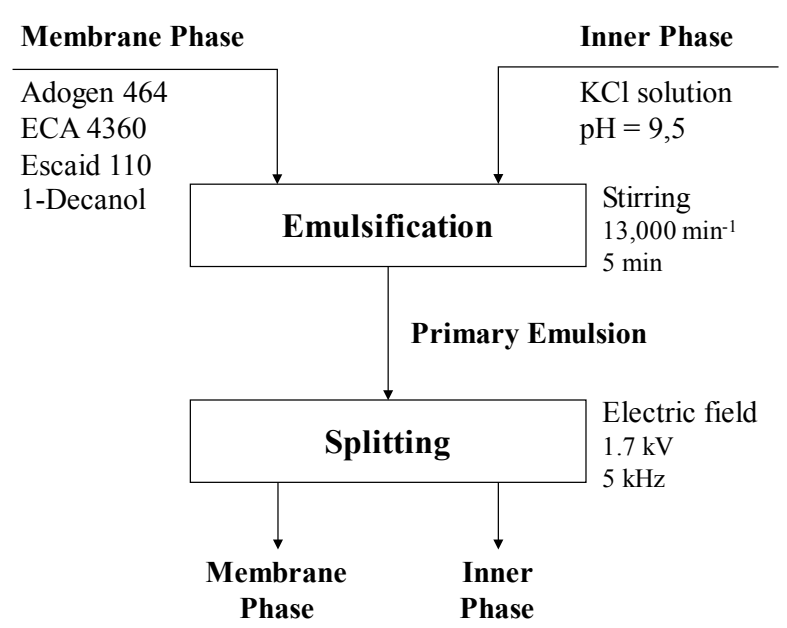

Figure 1. Schematic representation of the emulsification test methodology.

coalescence time in the electrostatic coalescer to facilitate the inner phase recovery.

\section{Results and Discussion}

Figure 2 shows the variation of the coalescence time as a function of the extractant concentration for different surfactant and modifier concentrations.

In order to discuss the obtained results, it is necessary to consider the behavior of a quaternary amine salt extractant (Adogen 464) in a low polarity diluent, such as Escaid 110.

Quaternary ammonium salts have a certain degree of polarity, with the molecular and ionic species coexisting. Therefore, their molecules are usually strongly associated in low-polarity and low-solvation solvents. This characteristic leads to the distribution of part of the extractant molecules on the $\mathrm{O} / \mathrm{W}$ interface and can act as a tensoactive agent. As a consequence, the additional improvement in the stability of the emulsions makes the droplet coalescence more difficult and increases the break-up time. This effect can be reduced by adding small quantities 


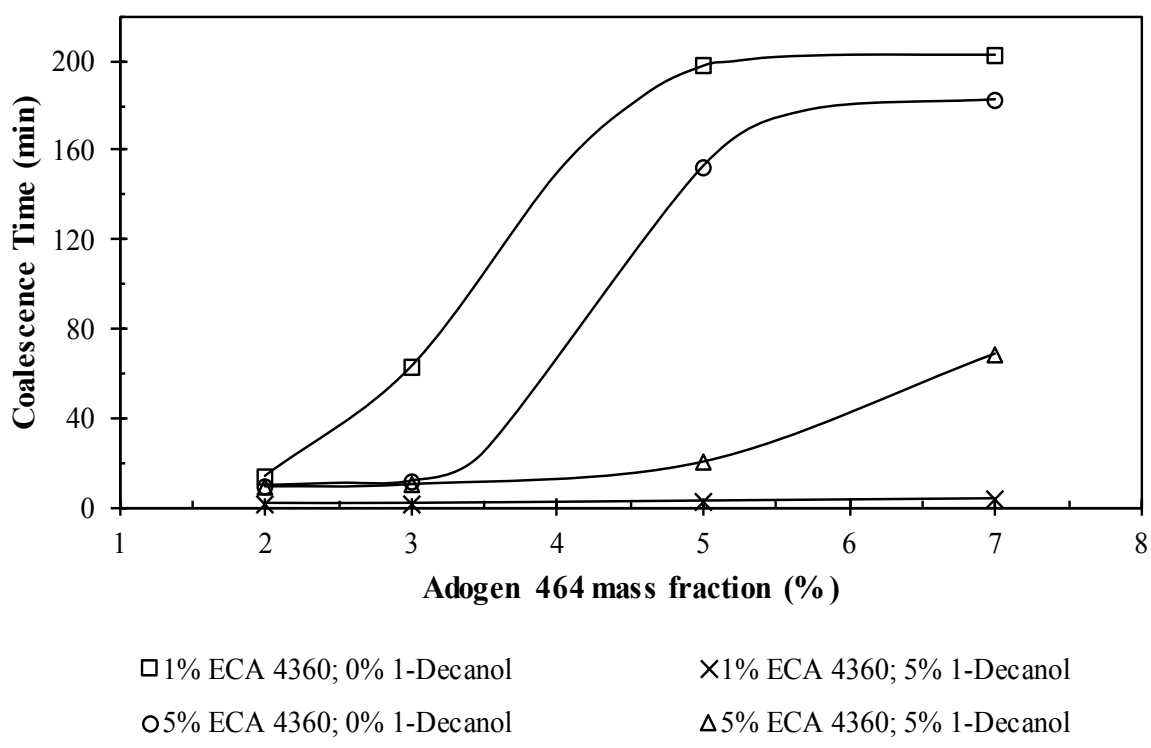

Figure 2. Influence of the extractant mass fraction on the coalescence time of the IP droplets for different surfactant and modifier concentrations. Conditions: stirring speed = $13,000 \mathrm{~min}^{-1}$, stirring time $=5 \mathrm{~min}, \mathrm{MP} / \mathrm{IP}=3 / 1,25^{\circ} \mathrm{C}$.

of additives which are soluble in both the extractant and the diluent, in order to modify their mutual solubility. Substances that present a large hydrocarbon portion, such as long-chain alcohols, are widely used for this purpose and act as a modifier [12] [14]. In this work, 1-decanol was used as a modifier.

It is shown, by the curves for the system without alcohol, presented in Figure 2 , that the increase in the extractant concentration results in longer coalescence times. Adogen 464 slightly increases the MP viscosity and probably also acts as a tensoactive agent, in synergy with surfactant ECA 4360, further increasing the system stability, and consequently the droplet coalescence time. This behavior highlights the importance of adding the modifier because in the extraction systems it is necessary that the extractant-solute complex leaves the interface and migrates into the membrane phase.

By analyzing the behavior of membranes without alcohol as a function of the surfactant concentration it is observed that the higher the concentration of ECA 4360 , the shorter the coalescence time, which is a non-expected result. This effect was more pronounced up to approximately $5 \%$ w/w Adogen 464 . Above such concentration, the coalescence times became closer. These results can be understood considering a similar effect described for alcohol in the literature [12] [14]. Since the surfactant is an amphiphilic molecule, as it is alcohol, it may increase the mutual solubility of the extractant and the diluent, even though it is not used in the system with this purpose. Therefore, the surfactant would leave the interface together with the extractant, thus decreasing the droplet coalescence time. However, for extractant concentrations higher than approximately $5 \% \mathrm{w} / \mathrm{w}$, the surfactant concentration becomes insufficient to improve the solvation of the extractant in the diluent, leading to an increased extractant concentration at the interface and, as a consequence, the effect of the surfactant con- 
centration on the coalescence time becomes less pronounced. A distinct effect was observed in the curves obtained at $1 \%$ and 5\% of ECA 4360 with $5 \%$ of 1-decanol. As expected, the presence of the modifier improved the mutual solubility of the extractant and the diluent. Then, the droplet coalescence time was higher for the highest surfactant concentration because the emulsifier remained available to stabilize the membrane.

When comparing the results of membrane phases without alcohol and with $5 \%$ of 1-decanol, it is observed a decrease in the droplet coalescence time with the use of this reagent, because there is probably a smaller amount of extractant at the $\mathrm{W} / \mathrm{O}$ interface due to the action of alcohol.

The variation of the droplet coalescence time as a function of the surfactant concentration for different extractant and modifier concentrations is shown in Figure 3.

As seen before, the droplet coalescence time decreased with the increase of the surfactant concentration in alcohol-free membranes. As previously proposed, the decrease in the membrane stability might be associated with the amphiphilic character of the surfactant, producing a similar, though less intense, effect than that of the alcohol (modifier), an improvement in the mutual solubility of the extractant and the diluent. In tests without the addition of alcohol, it was also observed that for the same amount of surfactant, the emulsion was more stable for the higher extractant concentrations. In this case, a higher extractant concentration implies a higher availability of this reagent in the W/O interface. This effect, associated with others such as the increase in the MP viscosity and the steric hindrance resulting from the large extractant molecule size and its high concentration, promotes an increase in the membrane stability. The initial increase of the curve for 5\% of Adogen 464 and $0 \%$ of 1-decanol can be explained

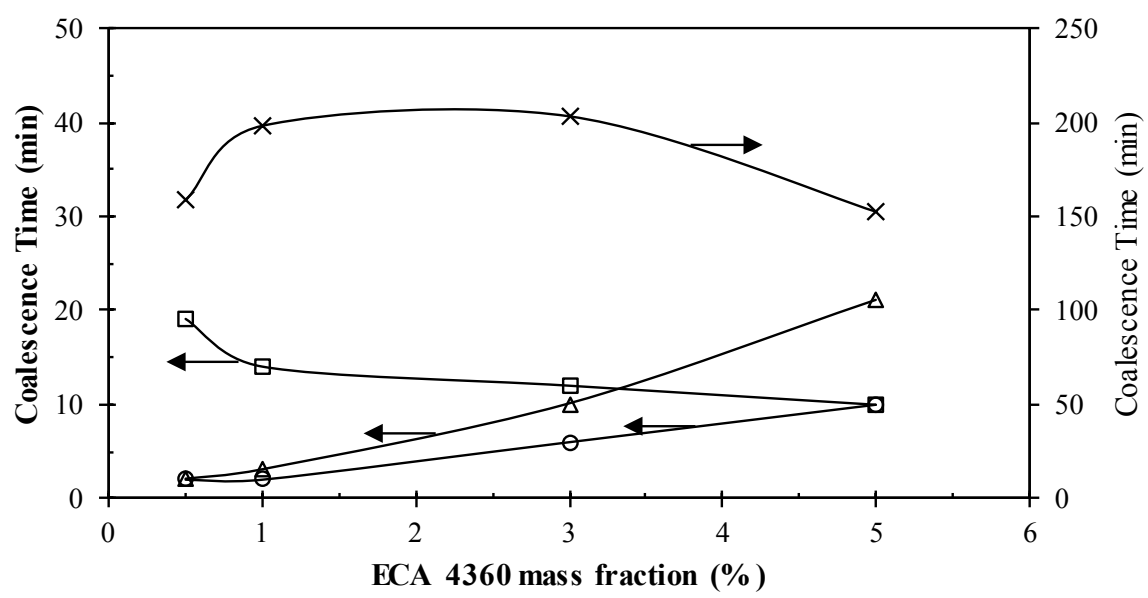

$\square 2 \%$ Adogen 464; 0\% 1-Decanol

$\Delta 5 \%$ Adogen $464 ; 5 \%$ 1-Decanol
O2\% Adogen 464; 5\% 1-Decanol

$\times 5 \%$ Adogen $464 ; 0 \%$ 1-Decanol

Figure 3. Influence of the surfactant concentration on the coalescence time of the IP droplets for different extractant and modifier concentrations. Conditions: stirring speed $=$ $13,000 \mathrm{~min}^{-1}$, stirring time $=5 \mathrm{~min}, \mathrm{MP} / \mathrm{IP}=3 / 1,25^{\circ} \mathrm{C}$. 
by the insufficient surfactant concentration to promote the effect previously described observed in the absence of the alcohol. Due to the greater amount of extractant present in the membrane phase, probably, a greater amount of surfactant is required to improve the solvation of the extractant in the diluent. Therefore, up to $3 \%$ of ECA 4360 , this reagent increases the membrane viscosity and its role is to increase the primary emulsion stability.

For membranes containing 5\% 1-decanol (curves for 2\% and 5\% of Adogen 464), the increase in the ECA 4360 concentration led to a higher coalescence time, which was even higher when a greater proportion of extractant was used, as previously proposed. Because of the presence of 1-decanol, the surfactant became more available to stabilize the emulsion at the interface, thus increasing the droplet coalescence time. When the amount of extractant is increased, there is one additional interfacial active agent to help stabilize the membrane. Consequently, the comparison of the curves for tests with and without alcohol for the same concentration of extractant (2\% Adogen 464 with $0 \%$ and 5\% 1-decanol, 5\% Adogen 464 with $0 \%$ and 5\% 1-decanol) reveals a convergence for each one of these pairs.

Figure 4 shows the variation of the coalescence time as a function of the concentration of the modifier for different extractant and surfactant concentrations.

The analysis of each pair of curves (1\% and 5\% of ECA 4360 with 5\% of Adogen 464 and 1\% and 5\% of ECA 4360 with $2 \%$ of Adogen 464) in the absence of alcohol (first points of the curves) shows that the system with a higher surfactant concentration presented a shorter droplet coalescence time, as seen before. These pairs also reveal that this behavior just becomes expressive at higher concentrations of extractant, as observed in the 5\% Adogen 464 curves. Under these conditions, the surfactant may be acting in a way similar to the modifier, as

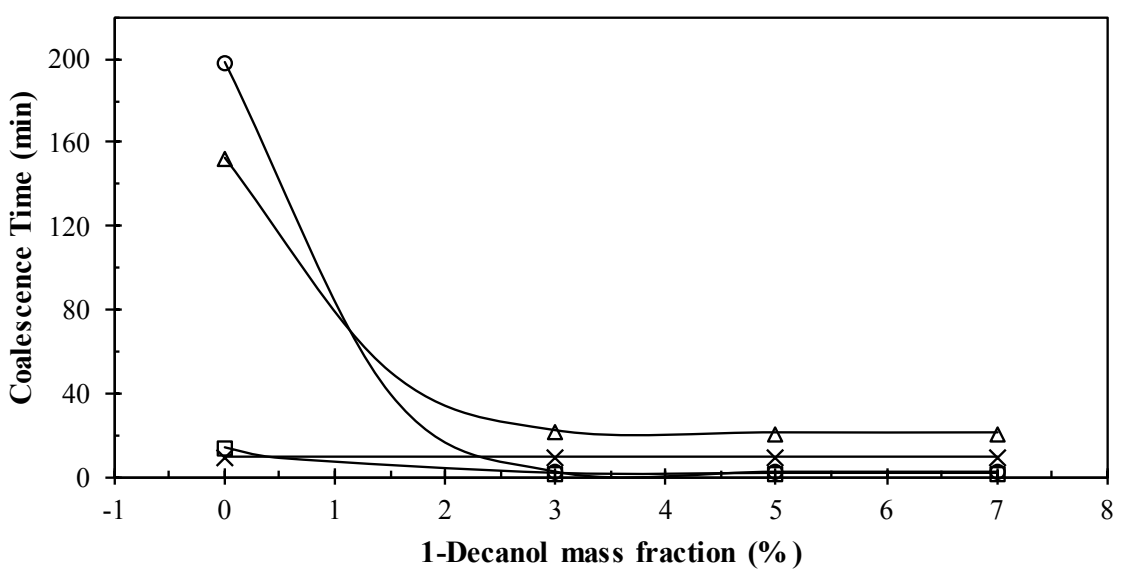

$$
\begin{aligned}
& \square 1 \% \text { ECA 4360; 2\% Adogen } 464 \\
& \times 5 \% \text { ECA 4360; 2\% Adogen } 464
\end{aligned}
$$

O 1\% ECA 4360; 5\% Adogen 464

$\triangle 5 \%$ ECA $4360 ; 5 \%$ Adogen 464

Figure 4. Influence of the modifier concentration on the coalescence time of the IP droplets for different surfactant and extractant concentrations. Conditions: stirring speed $=$ $13,000 \mathrm{~min}^{-1}$, stirring time $=5 \mathrm{~min}, \mathrm{MP} / \mathrm{IP}=3 / 1,25^{\circ} \mathrm{C}$. 
explained before. Additionally, the curves show that the membranes with 5\% of surfactant and $3 \%$ to $7 \%$ of alcohol present a higher droplet coalescence time than the ones with only $1 \%$ of surfactant for both concentrations of extractant investigated and for the same alcohol concentration range. As the purpose of the alcohol is to help solvating the extractant in the diluent, in its presence, the surfactant plays its main role, which is to stabilize the W/O interface.

Figure 4 also indicates that for all curves, excepting the one for 5\% of ECA 4360 with $2 \%$ of Adogen 464, the droplet coalescence time decreased with an increase in the concentration of 1-decanol up to $3 \%$. In the presence of the alcohol, probably the migration of the extractant from the W/O interface to the bulk of the membrane phase is easier. Then, the surfactant can act primarily as a tensoactive, stabilizing the primary emulsion. From $3 \% \mathrm{w} / \mathrm{w}$ of 1 -decanol, the droplet coalescence time does not vary anymore. Probably, an alcohol concentration of $3 \%$ is enough to allow the adequate solvation of the extractant in the diluent. Concerning the curve for 5\% of ECA 4360 with $2 \%$ of Adogen 464, the surfactant/extractant ratio was supposedly enough to allow the solvation of the extractant in the diluent, even in the absence of alcohol.

\section{Conclusions}

The emulsification tests conducted in order to evaluate the effect of the membrane phase composition on the stability of emulsions for the system studied have shown that it is necessary to use a modifier in order to improve the extractant solvation in the diluent because of the low solubility of quaternary ammonium salts in low polarity diluents. For 1-decanol, a concentration of $3 \%$ was sufficient to solvate Adogen 464 in Escaid 110. If no further alcohol is added, the surfactant, which has an amphiphilic character, acts as a modifier in the solvation of the extractant, decreasing its concentration at the W/O interface and reducing the stability of the emulsion formed. Additionally, the extractant itself acts as an interfacial active agent and increases the emulsion stability at higher concentrations in the MP and in the absence of the modifier. However, while the solvation of the extractant in the diluent improves with the addition of either the modifier or the surfactant, the emulsion stability diminishes due to the extractant removal from the interface.

Understanding the role of each component (extractant, surfactant and modifier) in the stability of the primary emulsion is very relevant for the efficient application of the LSM technique. It is important to emphasize that the role of each component in MP and their interactions fundamentally depend on the chosen composition and on the concentrations used, as they affect, significantly, the interfacial tension properties of the W/O interface.

\section{Acknowledgements}

The authors acknowledge CAPES, CNPq, FAPEMIG and PRPq by the financial support. 


\section{Conflicts of Interest}

The authors declare no conflicts of interest regarding the publication of this paper.

\section{References}

[1] Li, N. (1968) Separating Hydrocarbons with Liquid Membranes. US Patent $3,410,794$,

[2] Ma, H., Kökkılıç, O. and Waters, K.E. (2017) The Use of the Emulsion Liquid Membrane Technique to Remove Copper Ions from Aqueous Systems Using Statistical Experimental Design. Minerals Engineering, 107, 88-99.

https://doi.org/10.1016/j.mineng.2016.10.014

[3] Davoodi-Nasab, P., Rahbar-Kelishami, A., Safdari, J. and Abolghasemi, H. (2018) Evaluation of the Emulsion Liquid Membrane Performance on the Removal of Gadolinium from Acidic Solutions. Journal of Molecular Liquids, 262, 97-103. https://doi.org/10.1016/j.molliq.2018.04.062

[4] Zaheri, P. and Davarkhah, R. (2017) Rapid Removal of Uranium from Aqueous Solution by Emulsion Liquid Membrane Containing Thenoyltrifluoroacetone. Journal of Environmental Chemical Engineering, 5, 4064-4068.

https://doi.org/10.1016/j.jece.2017.07.076

[5] Konzen, C., Araújo, E.M., Balarini, J.C., Miranda, T.L.S. and Salum, A. (2014) Extraction of Citric Acid by Liquid Surfactant Membranes: Bench Experiments in Single and Multistage Operation. Chemical and Biochemical Engineering Quarterly Journal, 28, 289-299. https://doi.org/10.15255/CABEQ.2013.1789

[6] Othman, N., Noah, N.F.M., Shu, L.Y., Ooi, Z.-Y., Jusoh, N., Idroas, M., et al. (2017) Easy Removing of Phenol from Wastewater Using Vegetable Oil-Based Organic Solvent in Emulsion Liquid Membrane Process. Chinese Journal of Chemical Engineering, 25, 45-52. https://doi.org/10.1016/j.cjche.2016.06.002

[7] Draxler, J. and Marr, R. (1986) Emulsion Liquid Membranes Part I: Phenomenon and Industrial Application. Chemical Engineering and Processing. Process Intensification, 20, 319-329. https://doi.org/10.1016/0255-2701(86)80010-1

[8] Patnaik, P.R. (1995) Liquid Emulsion Membranes: Principles, Problems and Applications in Fermentation Processes. Biotechnology Advances, 13, 175-208. https://doi.org/10.1016/0734-9750(95)00001-7

[9] Salum, A. (1998) Estudo da Permeação de Cobalto e da Separação Cobalto/Níquel em Meio Sulfúrico, com Cyanex 272, pela Técnica de Membranas Líquidas Surfatantes. Doctorate Thesis, Federal University of Minas Gerais, Belo Horizonte. (In Portuguese)

[10] Rosen, M.J. and Kunjappu, J.T. (2012) Surfactants and Interfacial Phenomena. John Wiley \& Sons, New York. https://doi.org/10.1002/9781118228920

[11] Clayton, W. and Sumner, C.G. (1954) Clayton's the Theory of Emulsions and Their Technical Treatment. Chemical Publishing Company, London.

[12] Shimidt, V.S. (1971) Amine Extraction. Israel Program of Scientific Translations Ltd., Jerusalem.

[13] Konzen, C. (2000) Estudo da técnica de extração por membranas líquidas surfatantes aplicada à separação de ácido cítrico. Doctorate Thesis, Federal University of Minas Gerais, Belo Horizonte. (In Portuguese)

[14] Evangelista Jr., J.M.G. (2002) Estudo da extração seletiva de ferro, presente em 
efluentes clorídricos oriundos da decapagem de aços em usina siderúrgica, pela técnica de extração líquido-líquido. Doctorate Thesis, Federal University of Minas Gerais, Belo Horizonte. (In Portuguese)

\section{Nomenclature}

EP-external phase

IP-inner phase

LSM-liquid surfactant membranes

$\mathrm{MP}$-membrane phase

$\mathrm{O}-$ organic phase

$\mathrm{W}-$ aqueous phase 